\title{
Accumulation of Colloidal Particles in Flow Junctions Induced by Fluid Flow and Diffusiophoresis
}

\author{
Sangwoo Shin, ${ }^{1, *}$ Jesse T. Ault, ${ }^{2}$ Patrick B. Warren, ${ }^{3}$ and Howard A. Stone ${ }^{4}$ \\ ${ }^{1}$ Department of Mechanical Engineering, University of Hawaii at Manoa, Honolulu, Hawaii 96822, USA \\ ${ }^{2}$ Computational Sciences and Engineering Division, Oak Ridge National Laboratory, \\ Oak Ridge, Tennessee 37831, USA \\ ${ }^{3}$ Unilever R\&D Port Sunlight, Bebington, Wirral CH63 3JW, United Kingdom \\ ${ }^{4}$ Department of Mechanical and Aerospace Engineering, Princeton University, \\ Princeton, New Jersey 08544, USA
}

(Received 24 July 2017; revised manuscript received 24 September 2017; published 16 November 2017)

\begin{abstract}
The flow of solutions containing solutes and colloidal particles in porous media is widely found in systems including underground aquifers, hydraulic fractures, estuarine or coastal habitats, water filtration systems, etc. In such systems, solute gradients occur when there is a local change in the solute concentration. While the effects of solute gradients have been found to be important for many applications, we observe an unexpected colloidal behavior in porous media driven by the combination of solute gradients and the fluid flow. When two flows with different solute concentrations are in contact near a junction, a sharp solute gradient is formed at the interface, which may allow strong diffusiophoresis of the particles directed against the flow. Consequently, the particles accumulate near the pore entrance, rapidly approaching the packing limit. These colloidal dynamics have important implications for the clogging of a porous medium, where particles that are orders of magnitude smaller than the pore width can accumulate and block the pores within a short period of time. We also show that this effect can be exploited as a useful tool for preconcentrating biomolecules for rapid bioassays.
\end{abstract}

DOI: 10.1103/PhysRevX.7.041038

Subject Areas: Fluid Dynamics, Soft Matter

In flow channels, suspended particles free of external forces are typically expected to advect along with the fluid flow unless the particles are large enough to physically clog the channel or sticky enough to adhere to boundaries. However, recent observations suggest that this may not always be the case; particles that are less dense than the surrounding fluid may be trapped in flow junctions via the vortex breakdown phenomenon [1-3]. Such an unexpected trapping of particles in simple flow geometries implies unrecognized ways that flow systems can fail or respond in unusual ways.

Here, we report a chemically mediated particle trapping mechanism in flow junctions that is nearly insensitive to the particle size or density for colloidal materials. The observed particle trapping takes place when solute concentration gradients are present in the channel, for example, in the case of a pore connecting two streams carrying different solute concentrations. Near the flow junction, because of the advection, the solute concentration gradient can be very sharp, as sketched in Fig. 1(a). Such a

\footnotetext{
*sangwoos@hawaii.edu
}

Published by the American Physical Society under the terms of the Creative Commons Attribution 4.0 International license. Further distribution of this work must maintain attribution to the author(s) and the published article's title, journal citation, and DOI. steep gradient provides a suitable condition for colloidal particles to undergo directed motions such as diffusiophoresis $[4,5]$.

Diffusiophoresis, which has received recent interest because of experimental and theoretical advances [7-17], refers to the motion of colloidal particles induced by the solute gradients. The local solute gradient gives rise to the particle motion due to the osmotic pressure gradient developed along the particle surface (chemiphoresis) and the liquid junction potential generated by the diffusion of ions with different diffusivities (electrophoresis). The particle diffusiophoretic velocity $\boldsymbol{u}_{p}$, relative to the local flow velocity, is given as $\boldsymbol{u}_{p}=\Gamma_{p} \nabla \ln c$, where $\Gamma_{p}$ is the diffusiophoretic mobility and $c$ is the solute concentration [4,5]. The sharp solute gradient near the flow junction may drive particle motions against the flow direction depending on the sign of $\Gamma_{p}$ and the solute gradient. Furthermore, when the fluid velocity $\boldsymbol{u}_{f}$ and the diffusiophoretic velocity $\boldsymbol{u}_{p}$ have comparable magnitudes and are in opposite directions, particles may experience zero net velocity and accumulate in the region of the junction [see the inset of Fig. 1(a)]. Accumulation of colloidal particles using two opposing forces in a linear setting has been demonstrated previously; examples include thermophoresis versus diffusiophoresis [18], thermophoresis versus fluid flow [19], electrophoresis versus fluid flow [20], etc. The current study focuses on the fact that the fluid flow at channel junctions sharpens the 
(a)

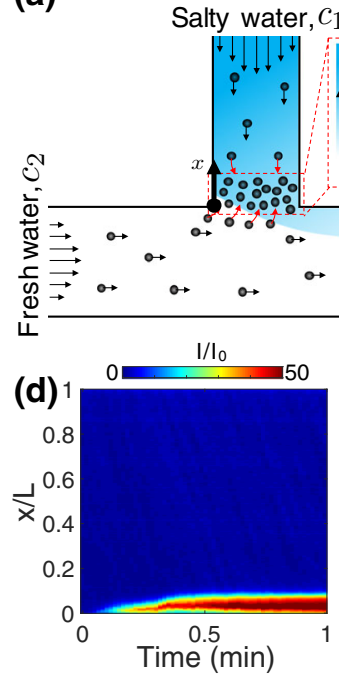

(b)

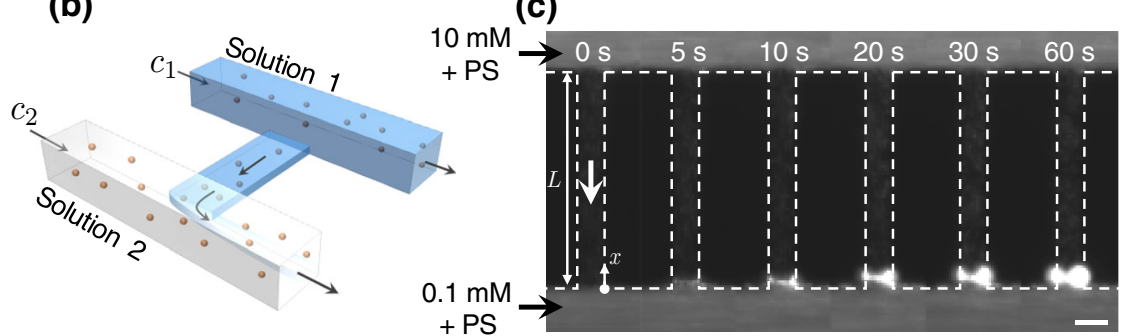

FIG. 1. Merging of two flow streams with different solute concentrations can lead to rapid accumulation of colloidal particles. (a) Particle accumulation near a flow junction due to the balance between diffusiophoretic motion (DP) and the fluid flow. (b) Schematic of the microfluidic channel for testing flow-induced diffusiophoretic particle accumulation. Pressure $p$ in the upper channel (solution 1) is larger than in the lower channel (solution 2), so solution 1 permeates through the pore and merges with solution 2. (c) Fluorescence image sequence of the particles [polystyrene (PS), diameter $=0.5 \mu \mathrm{m}$ ] accumulating near the bottom flow junction (Movie 1 in Ref. [6]). The solute $(\mathrm{NaCl})$ concentration in the upper stream is $c_{1}=10 \mathrm{mM}$, whereas the lower stream has $c_{2}=0.1 \mathrm{mM}$. The length of the pore is $L=400 \mu \mathrm{m}$. (d) Spatiotemporal plot of the width-averaged fluorescence intensity within the pore. (e) Evolution of the local maximum fluorescence intensity normalized by the intensity at $t=0 \mathrm{~s}\left(I / I_{0}\right)$. (f) Bright-field imaging of the particle accumulation near the flow junction (Movie 2 in Ref. [6]). Densely packed colloidal clusters are clearly seen after 1 minute of the experiment. All scale bars are $50 \mu \mathrm{m}$.

solute concentration profile, giving rise to a strong particle diffusiophoresis regardless of the flow velocity.

To demonstrate this flow-induced diffusiophoretic focusing effect in flow junctions, we use a microfluidic channel in which two large flow channels are connected by a narrow pore, as shown in Fig. 1(b) (see Materials and Methods for more details). The main channels are $100 \mu \mathrm{m}$ high, and the pore is $10 \mu \mathrm{m}$ high. This design allows precise control of the fluid permeation across the narrow pore because of its large flow resistance.

When colloidal suspensions with different solute concentrations $\left(c_{1}=0.1 \mathrm{mM}\right.$ and $\left.c_{2}=10 \mathrm{mM} \mathrm{NaCl}\right)$ are injected into the separate main channels, the colloidal particles (polystyrene, diameter $=0.5 \mu \mathrm{m}$ ) rapidly accumulate near the flow junction [Fig. 1(c) and Movie 1 in Ref. [6]). The spatiotemporal distribution of the fluorescence intensity shows that the particle accumulation is highly localized and stable [Fig. 1(d)]. As measured from the fluorescence intensity, the particle number density increases linearly until it slows down after a few minutes [Fig. 1(e)]. Assuming that the particle number density is linearly proportional to the fluorescence intensity [21], the local particle accumulation ratio is increased by a factor of 7000. This result indicates that beginning with a low volume fraction of colloids (here $0.01 \%$ ), the accumulated particles can locally reach a volume fraction of nearly $70 \%$, approaching the maximum packing limit, which is approximately $74 \%$ for monodisperse spheres. An accumulation of particles is also observed in Fig. 1(f) and Movie 2 in
Ref. [6], where the bright-field imaging reveals the densely clustered particles, which are otherwise difficult to visualize because of the low initial concentration and small size of the particles.

To gain insight on the observed flow-induced particle diffusiophoresis and accumulation, we consider a onedimensional pore having both a solute concentration gradient and fluid flow. In a semi-infinite pore, where the inside $(x \rightarrow \infty)$ concentration is $c_{1}$ and the outside ( $x=0)$ concentration is $c_{2}$ [Fig. 1(a)] and the fluid flow towards the outside is $u_{f}$, the steady-state solute concentration profile is $c(x)=c_{1}+\left(c_{2}-c_{1}\right) e^{-x u_{f} / D_{s}}$, where $D_{s}$ is the solute diffusion coefficient. Then, the position of the particles is in balance when the diffusiophoretic speed $u_{p}$ is equal to the flow speed $u_{f}$, i.e., $\Gamma_{p} \mathrm{~d} \ln c / \mathrm{d} x=u_{f}$. The balance condition yields the steady-state location $x_{0}$ of the colloidal particles, which is given by

$$
x_{0}=\frac{D_{s}}{u_{f}} \ln \left[\left(1-\frac{c_{2}}{c_{1}}\right)\left(1+\frac{\Gamma_{p}}{D_{s}}\right)\right] .
$$

Using experimental values $\left(D_{s}=1600 \mu \mathrm{m}^{2} / \mathrm{s}, \quad \Gamma_{p}=\right.$ $760 \mu \mathrm{m}^{2} / \mathrm{s}, u_{f}=25 \mu \mathrm{m} / \mathrm{s}$, and $\left.c_{2} / c_{1}=0.01\right)$, Eq. (1) yields $x_{0} \approx 25 \mu \mathrm{m}$, which agrees with the results shown in Fig. 1; from Fig. $1(\mathrm{~d}), x_{0} / L \approx 0.05$; thus, with $L=400 \mu \mathrm{m}, x_{0} \approx 20 \mu \mathrm{m}$.

Under typical conditions where $c_{1} / c_{2}<1$ and $\Gamma_{p} / D_{s}<1$, Eq. (1) implies that the main positional 


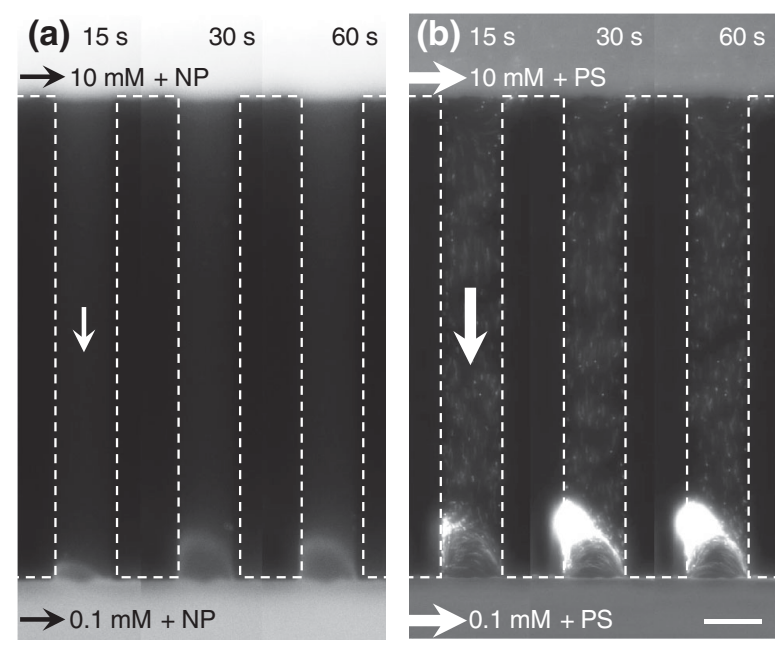

FIG. 2. Flow-induced diffusiophoretic accumulation occurs regardless of the particle size or the flow speed. Image sequences of (a) polystyrene nanoparticles $(\mathrm{NP}$, diameter $=50 \mathrm{~nm}$ ) focusing near the flow junction and (b) polystyrene particles (diameter $=0.5 \mu \mathrm{m}$ ) focusing near the flow junction at a higher pore flow speed $u_{f}$. In both cases, the solute concentration in the upper stream is $c_{1}=10 \mathrm{mM}$, and the solute concentration in the lower stream is $c_{2}=0.1 \mathrm{mM}$. The scale bar is $50 \mu \mathrm{m}$.

dependence of $x_{0}$ is on $D_{s} / u_{f}$, and there is only a rather weak dependence on the nature of the particles, which is expressed by $\Gamma_{p}$. In addition, the sign of $x_{0}$, which indicates the conditions under which particles accumulate in the pore $\left(x_{0}>0\right)$, is not affected by the flow speed, indicating that particle accumulation should be observed as long as the cross flow in the main channel is fast enough to maintain a constant solute concentration near the pore entrance. This independence is due to the fact that a faster outflow velocity leads to a steeper solute concentration gradient, in turn increasing the diffusiophoretic velocity of the particles and pulling them more strongly into the pore.

These arguments are further confirmed in Fig. 2, where the particles with smaller $\Gamma_{p}$ [polystyrene nanoparticles, diameter $=50 \mathrm{~nm}, \Gamma_{p}=301 \mu \mathrm{m}^{2} / \mathrm{s}$, Fig. 2(a)] or faster pore flow speed $u_{f}$ [Fig. 2(b)] can be captured effectively near the pore entrance, which illustrates that the particle accumulation in flow junctions can occur under broad conditions irrespective of the flow velocity or particle type. Notice that for the nanoparticles, although the particle accumulation still takes place, the magnitude of the accumulation is weaker than for the larger particles because of small $\Gamma_{p}$ and large diffusivity.

We notice that $u_{f}$ does not change significantly as the particles accumulate within the pore, allowing the particle accumulation location $x_{0}$ to be stable over time. The subtlety lies in the nature of diffusiophoretic flow through the dense plug of particles. Recall that $x_{0}$ arises because the diffusiophoretic speed $u_{p}$ balances the convection speed $u_{f}$. What this means is that, while a particle at this point is
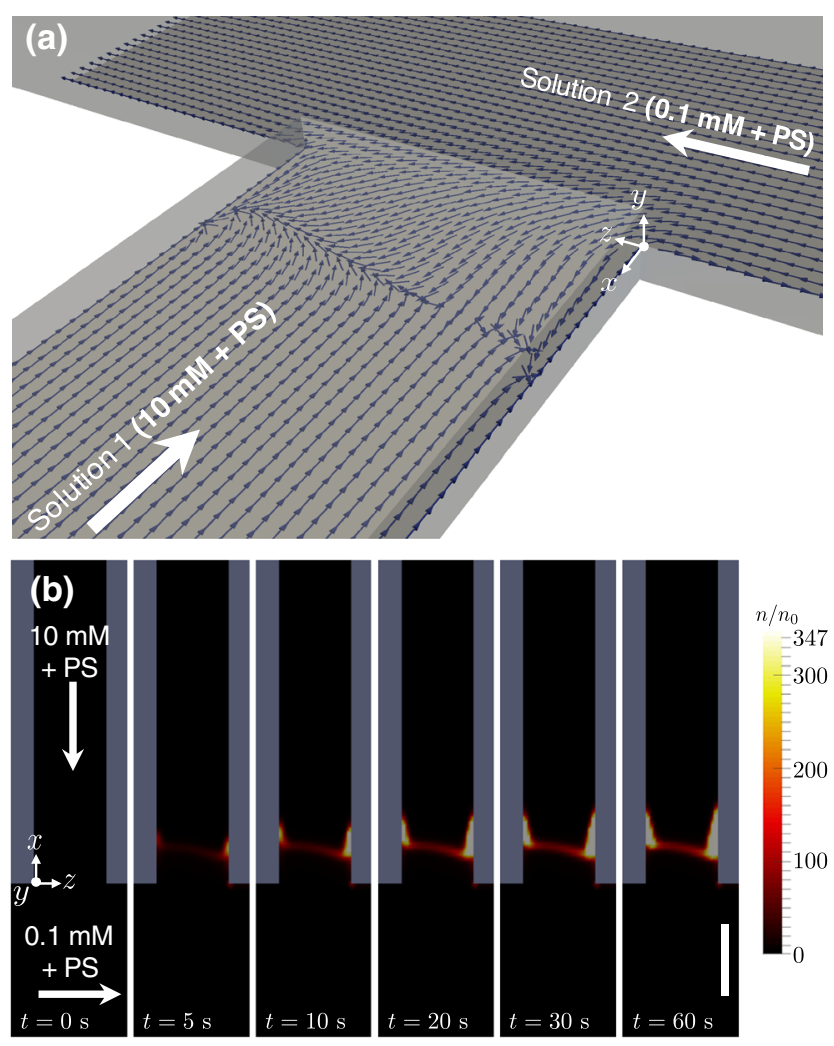

FIG. 3. 3D numerical simulations of the diffusiophoretic particle dynamics. (a) Particle trajectories on a slice taken through the center of the pore. Particles approach a position within the pore where fluid velocities are balanced by diffusiophoretic velocities. Ultimately, particles travel towards two accumulation points near the sides of the pore. (b) Computed particle concentrations normalized by the initial concentration $\left(n / n_{0}\right)$ over time, showing accumulation near the stable points seen in panel (a). Large white arrows indicate the mean flow direction. The scale bar is $50 \mu \mathrm{m}$.

stationary in the lab frame of reference, it is "slipping" at a speed $u_{p}$ through the fluid. This leads to an $r^{-3}$ perturbation to the flow field, not $r^{-1}$ as would happen if an external force were holding the particle in place, where $r$ is the distance away from the particle. As a consequence, corrections due to hydrodynamic interactions are absent in diffusiophoresis [5]. This implies that although a dense plug of particles forms, the fluid slips between them at a speed $u_{p}$, and the actual flow field is only mildly perturbed, in complete contrast to a plug formed by aggregated particles stuck to the wall, as will be shown in Fig. 4.

More detailed insights into the diffusiophoretic particle dynamics can be obtained by performing full 3D numerical simulations of the Navier-Stokes equations coupled with the solute and particle advection-diffusion equations and applying the diffusioosmotic wall slip flow conditions (see Materials and Methods for more details) [12,17]. Computed particle trajectories near the pore entrance are shown on a slice through the pore center plane in Fig. 3(a). 
The trajectories approach an approximately fixed depth in the pore where advection velocities balance the diffusiophoretic contribution. Because of the circulating flow that diverges from the center toward the wall, which is induced by the diffusioosmotic flow along the charged channel wall $[12,17]$, the particles are attracted toward the channel wall, leading to two distinct focusing locations. However, the crossflow brings asymmetry to the particle motion, and the particles are more likely to focus on the downstream side of the wall (right-hand side). This result occurs because the particles are also advected toward that location by the crossflow unless the crossflow is so strong as to sweep away the particles. The consequence of fast crossflow speed is that the strong shear flow dominates over diffusiophoresis and carries away the particles so that the focusing does not happen near the downstream side of the pore wall (right-hand side). However, there is still a strong particle accumulation near the upstream side of the pore wall (left-hand side) because this area is less influenced by the crossflow; this is shown in Fig. 2(b), where the cross flow speed $(14.6 \mathrm{~mm} / \mathrm{s})$ is approximately 16 times faster than the usual conditions $(0.9 \mathrm{~mm} / \mathrm{s})$.

Normalized time-dependent particle concentrations $n / n_{0}$ are shown in Fig. 3(b), assuming an initially uniform particle distribution at $n=n_{0}$. As can be seen, particles rapidly accumulate near the stable points at $x_{0} \approx 25 \mu \mathrm{m}$, which coincides with the experimental observations in Fig. 1.

The rapid clustering of colloidal particles near the flow junction has implications for the clogging of porous media for the unexpected conditions in which the particles are at low concentrations and are orders of magnitude smaller than the width of the pore; nevertheless, the particles can accumulate to block the pores. Typically, pore clogging is understood to be most likely to occur when the size of the particles is comparable to the pore size, such that steric effects like sieving or bridging can induce pore clogging [22]. In contrast, particles that are much smaller than the size of the pore are able to clog the pore only via particle aggregation, which is a much slower process than clogging driven by steric effects [22,23].

However, as shown in Fig. 4 (Movie 3 in Ref. [6]), where the main channels are now connected with multiple pores mimicking a porous medium, the flow-induced diffusiophoretic accumulation significantly enhances the particle aggregation, leading to rapid particle buildup near the pore entrances. Here, the solute concentration of the higher side $\left(c_{2}\right)$ is increased to $100 \mathrm{mM}(\mathrm{NaCl})$ to screen out the Debye layer so that the particles can easily aggregate or adhere to the wall. This situation mimics realistic solute conditions in nature (e.g., brackish water, physiological fluids, etc.). Also, the particles are injected only through the lower solute concentration stream $\left(c_{1}=0 \mathrm{mM}\right)$. Even when the particle size is 2 orders of magnitude smaller than the width of the pore, the aggregated particles formed by the flowinduced colloidal clusters cover the pore mouths within

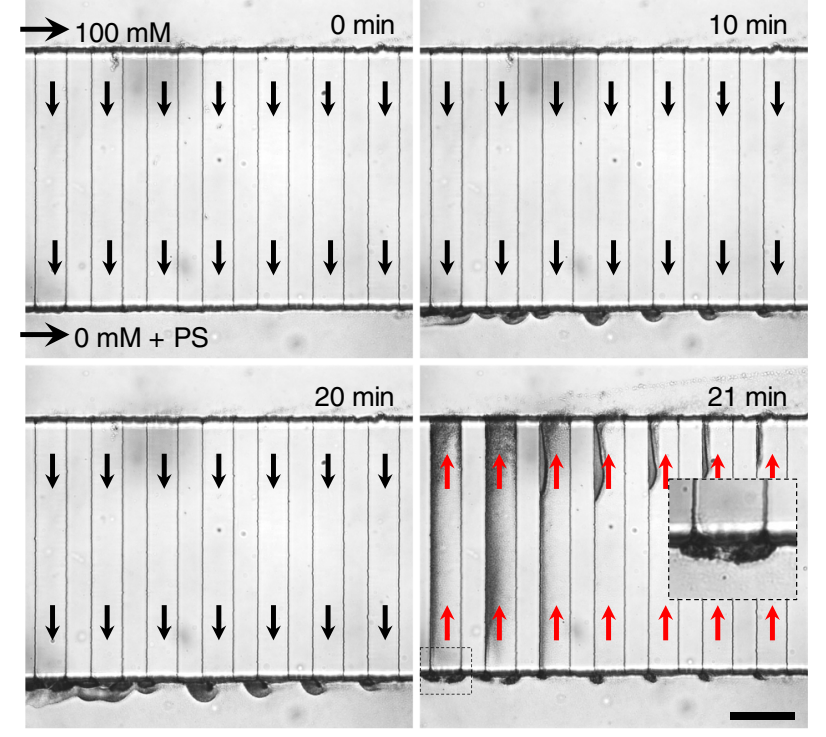

FIG. 4. Small particles can lead to irreversible clogging in large pores. Image sequence of small particles $(0.5 \mu \mathrm{m}$ in diameter $)$ accumulating near the large pore mouths $(50 \mu \mathrm{m}$ in width, see Movie 3 in Ref. [6]) due to the outflow of the upper concentrated stream. The solute concentration of the upper stream is $c_{1}=100 \mathrm{mM}$, and the solute concentration of the lower stream is $c_{2}=0 \mathrm{mM}$. Only the lower stream contains polystyrene particles. The bottom-right panel shows clogged particles near the pore mouths after the flow direction within the pores has been reversed. The inset shows a close-up image of the black dashed box. The scale bar is $100 \mu \mathrm{m}$.

10 minutes. Because of the aggregation, the majority of the particles remain even after the flow has changed directions, indicating irreversible clogging (bottom-right panel in Fig. 4 and Movie 3 in Ref. [6]). Fluid flow and solute gradients in porous media are commonly found in many natural environments, e.g., underground reservoirs [24] and estuaries [25], as well as artificial settings such as filtration membranes [26], thus indicating a variety of conditions where suspended particles may clog and alter the flow system.

While this flow-induced particle accumulation has potentially adverse effects, such as in the clogging of filtration membranes, this accumulation can also be exploited for practical applications. For example, because this effect can locally concentrate colloidal particles almost to their maximum packing limit, it could be used for preconcentrating or separating particles in a short period of time for various analytical purposes [21,27].

A conceptual schematic of an automated sample preconcentrator using the flow-induced accumulation effect is presented in Figs. 5(a)-5(c). In this setup, the pores act as a "trap" for the analytes. Upon initiation of the outflow through the pores, the dilute analytes are highly concentrated near the flow junctions, and detection or analysis can be made in this region, owing to the stable formation of the clusters [Fig. 5(a)]. If the analytes need to be mixed with some other chemicals for further analysis or require flow-based detection 

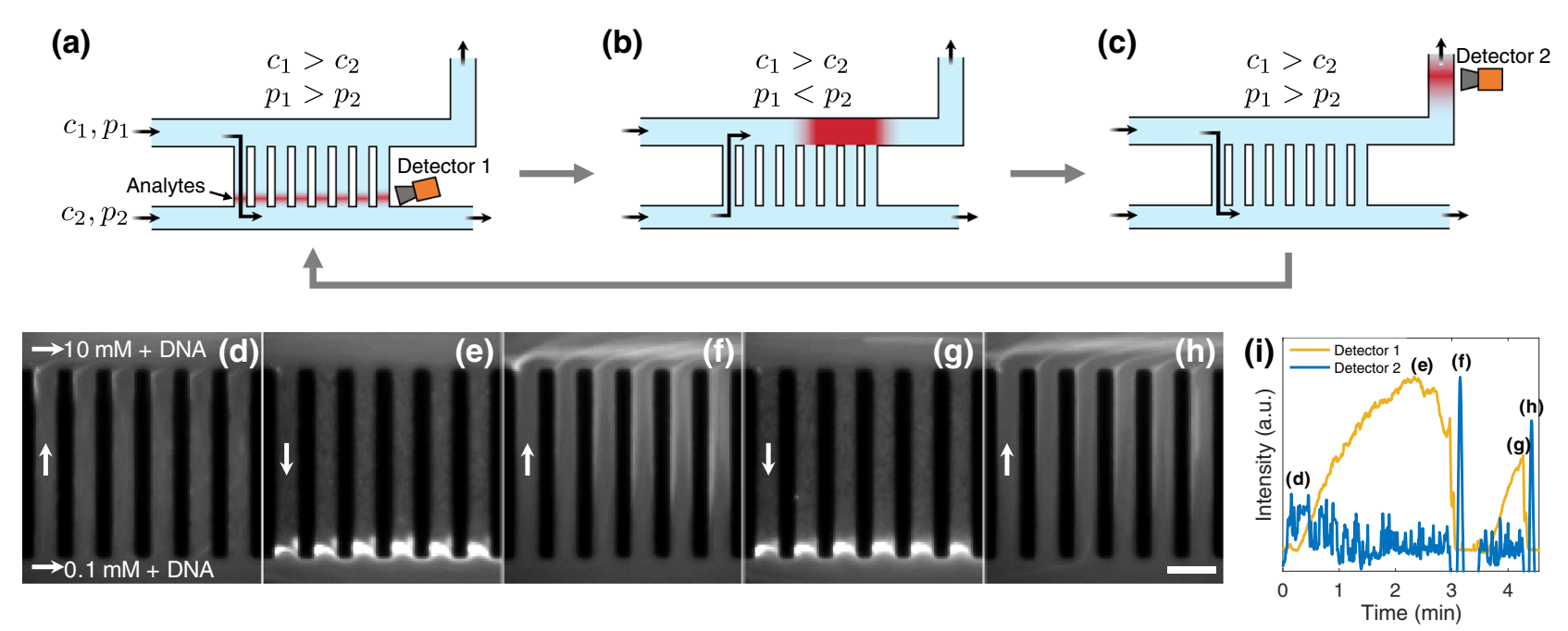

FIG. 5. Automated sample preconcentrator for a rapid bioassay. (a-c) A conceptual schematic of the preconcentrator using the flowinduced diffusiophoretic accumulation. An array of pores operates as a trap for the analytes. (a) Upon initiation of the outflow through the pores due to the higher pressure $p_{1}$ at the upper channel than at the lower channel $\left(p_{2}\right)$, the dilute analytes are rapidly concentrated near the flow junctions, and a stable detection can be made in this region. (b) The accumulated analytes can be transferred to the other side of the channel by changing the pressure difference across the pores for further mixing with some other chemicals or for flow-based detection. (c) Subsequently, the analytes can be analyzed at the downstream. $(\mathrm{d}-\mathrm{h})$ Experimental results showing fluorescence image sequence of preconcentration and transfer of $\lambda$-DNAs using the flow-induced diffusiophoretic accumulation (Movie 4 in Ref. [6]). Each image corresponds to the time indicated in panel (i). The solute concentration of the upper stream is $c_{1}=10 \mathrm{mM}$, and the solute concentration of the lower stream is $c_{2}=0.1 \mathrm{mM}$. Both streams contain $\lambda$-DNAs. (i) Fluorescence intensity measurement near the flow junction (orange curve) and downstream in the upper channel (blue curve). The scale bar in panel (h) is $100 \mu \mathrm{m}$.

such as flow cytometry, then the accumulated analytes can be easily transferred to the other side of the channel by changing the pressure difference across the pores [Fig. 5(b)]. Subsequently, the analytes can be mixed and analyzed at a downstream location [Fig. 5(c)].

As a demonstration, $\lambda$-DNAs are preconcentrated using the presented technique [Figs. 5(d)-5(i) and Movie 4 in Ref. [6]]. Here, the height of the channel is uniform everywhere $(10 \mu \mathrm{m})$. Because of the balance between the fluid flow and diffusiophoresis, the dilute DNA molecules accumulate near the flow junctions [Fig. 5(e)]. This accumulation occurs in a very stable manner, allowing easy detection of the analytes. The average intensity near the accumulated region is plotted over time in Fig. 5(i) (orange curve). The accumulated DNA molecules can be passed to the other side of the channel by increasing the pressure at the lower flow channel [Fig. 5(f)]; then, they can be detected downstream [blue curve in Fig. 5(i)]. The whole process can be repeated by reversing the pressure again [Figs. $5(\mathrm{~g})$ and $5(\mathrm{~h})$ ], allowing continuous and autonomous operation. While this demonstration is only a proof of concept, the device can be further optimized for practical applications. For instance, narrower main channels will help reduce the dead sample volume flowing in the main channels, and the captured sample volume can be substantially enhanced by massively increasing the number of pores.

By balancing fluid flow and diffusiophoresis, we have observed rapid and robust trapping of colloidal particles near a flow junction. The present mechanism can efficiently capture colloidal particles regardless of their density and size since the diffusiophoretic dynamics originate from the electrostatic interaction between the solute and the surface charge of the particles. The consequences of the particle accumulation can be significant in many porous systems having fluid flow and solute gradients since the particles, which are orders of magnitude smaller than the pore size, can rapidly block the pores, as shown in Fig. 4. Possible practical examples of this particle trapping include the formation of microbial biofilms and biofilm streamers in natural habitats such as springs and marshes where solutes and nutrients are abundant [28-30], microbial infections in the human body such as oral systems and gastrointestinal tracts [29], and fouling in reverse-osmosis systems due to ion-concentration polarization [31]. This mechanism for rapid particle accumulation also suggests a useful functionality, where the observed phenomenon can be used for collecting biomolecules for separation purposes or rapid bioassays.

\section{MATERIALS AND METHODS}

Materials.-Fluorescent polystyrene particles (diameter $=0.5 \mu \mathrm{m}$ ) were purchased from Bangs Laboratories, and $\lambda$-DNA was purchased from New England Biolabs. YOYO-1 was purchased from Life Technologies. Polydimethylsiloxane (PDMS) was purchased from Dow Corning (Sylgard 184). NaCl, Tris-HCl, and ethylenediaminetetraacetic acid (EDTA) were purchased from Sigma-Aldrich. 
Microfluidic experiments.-The microfluidic channels were made out of PDMS. The device has two straight main channels that are parallel to each other and pores that connect the two main channels (single pore for the experiments in Figs. 1 and 2, and multiple pores for the experiments in Figs. 4 and 5). The height of the pore is $10 \mu \mathrm{m}$, whereas the height of the main channels is $100 \mu \mathrm{m}$ except for the DNA experiments (Fig. 4) where the channel height is uniform everywhere $(10 \mu \mathrm{m})$. A computer-controlled pressure-driven pump (MFCS-EZ, Fluigent) was used to feed individual solutions through the main channels. The particles were visualized with an inverted fluorescence microscope (DMI4000B, Leica) and analyzed with ImageJ.

The $\lambda$-DNA was stained by adding 1 vol\% of YOYO- 1 dye to $50 \mu \mathrm{g} / \mathrm{ml}$ of $\lambda$-DNA in Tris-EDTA buffer $(10 \mathrm{mM}$ Tris- $\mathrm{HCl}, 1 \mathrm{mM}$ EDTA). After heating the solution at $50^{\circ} \mathrm{C}$ for 2 hours, the solution was diluted by 10 times with either $0.1 \mathrm{mM}$ or $10 \mathrm{mM} \mathrm{NaCl}$ solutions.

Numerical simulations.-Because of the presence of three-dimensional effects including diffusioosmotic wall slip velocities and non-negligible secondary fluid advection velocities, a full 3D numerical solver was developed that couples the solution of the Navier-Stokes equations with the diffusioosmotic wall slip velocity, as well as the solute and particle advection-diffusion equations [12,17]. The diffusioosmotic wall slip condition is a function of the wall surface charge and local solute concentrations, and the particle diffusiophoretic velocity contribution is also a function of the local solute concentration. The diffusioosmotic wall slip velocity is given as $\boldsymbol{u}_{w}=-\Gamma_{w} \nabla \ln c$, where $\Gamma_{w}$ is the diffusioosmotic mobility of the channel surface $\left(\Gamma_{w}=520 \mu \mathrm{m}^{2}\right.$ for polydimethylsiloxane in $10-\mathrm{mM} \mathrm{NaCl}$ solution at neutral $\mathrm{pH}$ [17]). The 3D numerical simulations were performed using a finite-volume solver based on the simpleFoam and scalarTransport Foam solvers of the OpenFOAM computation fluid dynamics library [32]. The geometry was chosen to exactly match the experiments (see Fig. 3).

For this problem, we assume only one-way coupling between the fluid-plus-solute and particle dynamics; i.e., we assume that the particle dynamics do not affect the flow nor the solute motions. We impose boundary conditions of no-slip velocity for the fluid at the walls, and no-penetration conditions for both the solute and particles at the walls. At the inlet to the pore, we impose a uniform velocity of $20 \mu \mathrm{m} / \mathrm{s}$ (as measured from the experiments), a uniform solute concentration of $10 \mathrm{mM}(\mathrm{NaCl})$, and a zero normal gradient boundary condition on the pressure. At the inlet to the main channel, we impose a uniform fluid velocity of $1 \mathrm{~mm} / \mathrm{s}$, a uniform solute concentration of $0.1 \mathrm{mM}$, and a zero normal pressure gradient boundary condition for the pressure. We let the initial particle concentration be uniform everywhere. At the outlet, we impose a zero normal gradient boundary condition on the velocity, solute concentration, and particle concentration, which assumes that the flow is fully developed. We set the pressure at the outlet to zero. This combination of pressure boundary conditions works very well, except for within a couple of grid cells of the inlets, with negligible effects on the dynamics of interest.

Since we have assumed one-way coupling on the particle dynamics, and the fluid and solute concentration boundary conditions are steady in time, we expect them to evolve towards a steady state as long as the Reynolds number remains small enough. Because we are primarily interested in visualizing the particle dynamics, we first solve for the steady-state velocity and solute concentration solution, and we use this result to iterate the particle dynamics. Thus, we first solve the advection-diffusion equation for the solute while simultaneously relaxing the fluid velocity using the SIMPLE algorithm of the simpleFoam solver. For this procedure, we use initial conditions of zero fluid velocity and a solute concentration of $0.1 \mathrm{mM}$ (equal to the boundary condition for concentration at the main inlet). We monitor the norm of the time derivative of the solute concentration field in order to measure convergence in time. Once we achieve the steady-state fluid-plus-solute profiles, we set an initial condition on the particle concentration to be uniform everywhere. We then use the background steady-state fluid-plus-solute profiles to update the particle concentration using the modified advection diffusion equation for the particles, which includes the diffusiophoretic contribution [17]. Solving forward in time, the particle concentrations also eventually approach a steady-state condition, with rapid particle accumulation in the pore. This approach is computationally efficient for getting a picture of the particle dynamics that is physically analogous to running the flow channel to the steady state and suddenly imposing uniform particle concentrations everywhere. For the purposes of visualizing the particle accumulation, and for calculating final steady-state velocity and concentration profiles, this approach is quite efficient. The fully coupled three-dimensional simulations provide strong agreement with the experimental results, validating the solver [17].

\section{ACKNOWLEDGMENTS}

We thank Sarangan Chari for stimulating discussions and Orest Shardt for kindly providing the image analysis code. We acknowledge Unilever Research and the Princeton Environmental Institute for support of this research. We thank the NSF (CBET-1702693) for partial support of this research. P. B. W. discloses a substantive stock holding in Unilever PLC. This manuscript has been authored by UTBattelle, LLC under Contract No. DE-AC05-00OR22725 with the U.S. Department of Energy. The United States Government retains and the publisher, by accepting the article for publication, acknowledges that the United States Government retains a nonexclusive, paid-up, irrevocable, world-wide license to publish or reproduce the published 
form of this manuscript, or allow others to do so, for United States Government purposes. The Department of Energy will provide public access to these results of federally sponsored research in accordance with the DOE Public Access Plan [33]. Research sponsored by the Laboratory Directed Research and Development Program of Oak Ridge National Laboratory, managed by UT-Battelle, LLC, for the U.S. Department of Energy.

[1] D. Vigolo, S. Radl, and H. A. Stone, Unexpected Trapping of Particles at a T Junction, Proc. Natl. Acad. Sci. U.S.A. 111, 4770 (2014).

[2] S. Shin, J. T. Ault, and H. A. Stone, Flow-Driven Rapid Vesicle Fusion via Vortex Trapping, Langmuir 31, 7178 (2015).

[3] J. T. Ault, A. Fani, K. K. Chen, S. Shin, F. Gallaire, and H. A. Stone, Vortex-Breakdown-Induced Particle Capture in Branching Junctions, Phys. Rev. Lett. 117, 084501 (2016).

[4] D. C. Prieve, J. L. Anderson, J. P. Ebel, and M. E. Lowell, Motion of a Particle Generated by Chemical Gradients. Part 2. Electrolytes, J. Fluid Mech. 148, 247 (1984).

[5] J. L. Anderson, Colloid Transport by Interfacial Forces, Annu. Rev. Fluid Mech. 21, 61 (1989).

[6] See Supplemental Material at http://link.aps.org/ supplemental/10.1103/PhysRevX.7.041038 for details.

[7] B. Abécassis, C. Cottin-Bizonne, C. Ybert, A. Ajdari, and L. Bocquet, Boosting Migration of Large Particles by Solute Contrasts, Nat. Mater. 7, 785 (2008).

[8] J. Palacci, B. Abécassis, C. Cottin-Bizonne, C. Ybert, and L. Bocquet, Colloidal Motility and Pattern Formation under Rectified Diffusiophoresis, Phys. Rev. Lett. 104, 138302 (2010).

[9] J. F. Brady, Particle Motion Driven by Solute Gradients with Application to Autonomous Motion: Continuum and Colloidal Perspectives, J. Fluid Mech. 667, 216 (2011).

[10] D. Florea, S. Musa, J. M. R Huyghe, and H. M. Wyss, Long-Range Repulsion of Colloids Driven by Ion Exchange and Diffusiophoresis, Proc. Natl. Acad. Sci. U.S.A. 111, 6554 (2014).

[11] A. Kar, T.-Y. Chiang, I. O. Rivera, A. Sen, and D. Velegol, Enhanced Transport into and out of Dead-End Pores, ACS Nano 9, 746 (2015).

[12] S. Shin, E. Um, B. Sabass, J. T. Ault, M. Rahimi, P. B. Warren, and H. A. Stone, Size-Dependent Control of Colloid Transport via Solute Gradients in Dead-End Channels, Proc. Natl. Acad. Sci. U.S.A. 113, 257 (2016).

[13] A. Banerjee, I. Williams, R. N. Azevedo, M. E. Helgeson, and T. M. Squires, Soluto-Inertial Phenomena: Designing Long-Range, Long-Lasting, Surface-Specific Interactions in Suspensions, Proc. Natl. Acad. Sci. U.S.A. 113, 8612 (2016).

[14] N. Shi, R. Nery-Azevedo, A. I. Abdel-Fattah, and T. M. Squires, Diffusiophoretic Focusing of Suspended Colloids, Phys. Rev. Lett. 117, 258001 (2016).
[15] R. F. Stout and A. S. Khair, Influence of Ion Sterics on Diffusiophoresis and Electrophoresis in Concentrated Electrolytes, Phys. Rev. Fluids 2, 014201 (2017).

[16] S. Shin, O. Shardt, P. B. Warren, and H. A. Stone, Membraneless Water Filtration using $\mathrm{CO}_{2}$, Nat. Commun. 8, 15181 (2017).

[17] S. Shin, J. T. Ault, J. Feng, P. B. Warren, and H. A. Stone, Low-Cost Zeta Potentiometry Using Solute Gradients, Adv. Mater. 29, 1701516 (2017).

[18] Y. T. Maeda, A. Buguin, and A. Libchaber, Thermal Separation: Interplay between the Soret Effect and Entropic Force Gradient, Phys. Rev. Lett. 107, 038301 (2011).

[19] S. Duhr and D. Braun, Optothermal Molecule Trapping by Opposing Fluid Flow with Thermophoretic Drift, Phys. Rev. Lett. 97, 038103 (2006).

[20] D. Stein, Z. Deurvorst, F. H. J. van der Heyden, W. J. A. Koopmans, A. Gabel, and C. Dekker, Electrokinetic Concentration of DNA Polymers in Nanofluidic Channels, Nano Lett. 10, 765 (2010).

[21] B. Jung, R. Bharadwaj, and J. G. Santiago, Thousandfold Signal Increase Using Field-Amplified Sample Stacking for On-Chip Electrophoresis, Electrophoresis 24, 3476 (2003).

[22] E. Dressaire and A. Sauret, Clogging of Microfluidic Systems, Soft Matter 13, 37 (2017).

[23] H. M. Wyss, D. L. Blair, J. F. Morris, H. A. Stone, and D. A. Weitz, Mechanism for Clogging of Microchannels, Phys. Rev. E 74, 061402 (2006).

[24] W. Song, T. W. de Haas, H. Fadaei, and D. Sinton, Chip-offthe-Old-Rock: The Study of Reservoir-Relevant Geological Processes with Real-Rock Micromodels, Lab Chip 14, 4382 (2014).

[25] M. J. Kaiser, Marine Ecology: Processes, Systems, and Impacts (Oxford University Press, New York, 2011).

[26] S. S. Sablani, M. F. A. Goosen, R. Al-Belushi, and M. Wilf, Concentration Polarization in Ultrafiltration and Reverse Osmosis: A Critical Review, Desalination 141, 269 (2001).

[27] F. Kitagawa and K. Otsuka, Recent Applications of On-Line Sample Preconcentration Techniques in Capillary Electrophoresis, J. Chromatogr. A 1335, 43 (2014).

[28] P. R. Jones, M. T. Cottrell, D. L. Kirchman, and S. C. Dexter, Bacterial Community Structure of Biofilms on Artificial Surfaces in an Estuary, Microb. Ecol. 53, 153 (2007).

[29] I. Klapper and J. Dockery, Mathematical Description of Microbial Biofilms, SIAM Rev. 52, 221 (2010).

[30] K. Drescher, Y. Shen, B. L. Bassler, and H. A. Stone, Biofilm Streamers Cause Catastrophic Disruption of Flow with Consequences for Environmental and Medical Systems, Proc. Natl. Acad. Sci. U.S.A. 110, 4345 (2013).

[31] R. Guha, X. Shang, A. L. Zydney, D. Velegol, and M. Kumar, Diffusiophoresis Contributes Significantly to Colloidal Fouling in Low Salinity Reverse Osmosis Systems, J. Membr. Sci. 479, 67 (2015).

[32] H. G. Weller, G. Tabor, H. Jasak, and C. Fureby, A Tensorial Approach to Computational Continuum Mechanics Using Object-Oriented Techniques, Comput. Phys. 12, 620 (1998).

[33] http://energy.gov/downloads/doe-public-access-plan. 